\title{
Author Index to Volume 30
}

\begin{tabular}{|c|c|}
\hline Ajami A, 15 & Dalle-Feste C, 430 \\
\hline Alkan M, 304 & Daval J-L, 100 \\
\hline Alpan $G, 616$ & Davis $\mathrm{PB}, 75$ \\
\hline Antigua $\mathrm{MC}, 522$ & De Abreu R, 550 \\
\hline Aperia A, 131 & Decena JA, 202 \\
\hline Aranda JV, 106 & DeGoede J, 491 \\
\hline Avery ME, 299 & de Groot CJ, 34 \\
\hline Aw TY, 112 & Denne SC, 23,423 \\
\hline Baan J, 375 & Deoras KS, 170 \\
\hline Badsberg JH, 464 & DeSouza MM, 154 \\
\hline Bain MD, 560 & De Vivo DC, 1 \\
\hline Ball $\mathrm{KT}, 513$ & Dianzani I, 435 \\
\hline Barendregt LG, 487 & Diven W, 146 \\
\hline Barna $T, 106$ & Dombrowski Jr GJ, 331 \\
\hline Barnes KD, 50 & Dowton SB, 444 \\
\hline Barshop B, 322 & D’Souza SW, 541 \\
\hline Barshop BA, 15 & Dudman NPB, 544 \\
\hline Beharry K, 106 & Edberg K-E, 181, 496, 501 \\
\hline Benton W, 181 & Edelson PJ, 118 \\
\hline Berkenbosch A, 491 & Ekström-Jodal B, 496, 501 \\
\hline Bernstein IM, 154 & Engström E, 150 \\
\hline Bertorello A, 131 & Epley M, 146 \\
\hline Bland R, 616 & Eriksson UJ, 150 \\
\hline Blei F, 266 & Ervin $\mathrm{MG}, 45$ \\
\hline Block SM, 50 & Everett AD, 252 \\
\hline Boekkooi PF, 479 & \\
\hline Bongers-Schokking CJ, 34 & Farrar MA, 610 \\
\hline Bossi E, 574 & Faucher DJ, 368 \\
\hline Bradley LM, 261 & Felix EA, 135 \\
\hline Bristow J, 381 & Fernhoff PM, 1 \\
\hline Brook CGD, 276 & Ferraris S, 435 \\
\hline Browne LE, 457 & Ferrero GB, 435 \\
\hline Brusilow SW, 622 & Fink I, 597 \\
\hline Buescher ES, 135 & Fisher DA, 28, 45 \\
\hline Buja LM, 610 & Folbergrová J, 473 \\
\hline Burdach S, 270 & Fomon SJ, 391 \\
\hline Burri R, 574 & Foreman JW, 528 \\
\hline Buts JP, 286 & Frank L, 413, 522 \\
\hline Cairo MS, 362,554 & $\begin{array}{l}\text { Frenck RW, } 135 \\
\text { Friedman WF } 83\end{array}$ \\
\hline Calder AG, 418 & Fukao T, 439 \\
\hline Carlson SE, 404 & Fukuda Y, 131 \\
\hline Castro R, 45 & Fuse $Y, 28$ \\
\hline Cha C-JM, 40 & Gall DG 342 \\
\hline Chacko A, 124 & Gall DG, 342 \\
\hline Chalmers RA, 560 & Ganzuka M, 239 \\
\hline Chan JCM, 528 & Garcia D, 579 \\
\hline Chemtob S, 106 & Gargus JJ, 216 \\
\hline Chen F, 83, 89 & Garlick PJ, 418 \\
\hline Cheng PY, 202 & Gauldin HE, 509 \\
\hline Cheng Y, 202 & Gelardi NL, 40 \\
\hline Chevalier RL, 252 & Gilbert T, 450 \\
\hline Chung Y, 75 & Gluckman PD, 513 \\
\hline Clark S, 554 & Goldstein RE, 261 \\
\hline Clyman R, 616 & Gomez RA, 252 \\
\hline Coates PM, 211 & Götze B, 69 \\
\hline Coehlo Jr AM, 534 & Granoff DM, 124 \\
\hline Collette E, 286 & Greco A, 266 \\
\hline Colon EJ, 34 & Greisen G, 570 \\
\hline Connuck DM, 509 & Grossman G, 239 \\
\hline Cooke RE, 624 & Guardamagna $O, 435$ \\
\hline Cooke RJ, 404 & Gunn TR, 513 \\
\hline Copeland KC, 154 & Guthrie RD, 606 \\
\hline Corbet A, 190 & Haglund $\AA, 150$ \\
\hline Corbett RJT, 579 & Hale DE, 211 \\
\hline Cornelissen M, 550 & Hansen JC, 351 \\
\hline Cotton RGH, 435 & Hasan SU, 193 \\
\hline Crain-Denoyelle A-M, 290 & $\begin{array}{l}\text { Masan SU, } 193 \\
\text { Hashimoto T, } 439\end{array}$ \\
\hline Curstedt T, 69, 591 & Herschkowitz N 574 \\
\hline Czaja JF, 261 & Heyman M, 290 \\
\hline Dabbagh $S, 146$ & Heymann MA, 62 \\
\hline
\end{tabular}

Ajami A, 15

Avery ME, 299

Aw TY, 112

Baan J, 37

Barendregt LG, 487

Barshop BA, 15

Beharry K, 106

Berkenbosch A, 49

Bernstein IM, 154

Blei F, 266

Block SiV

Bossi E 574

Bradley LM, 26

Bristow J, 381

Browne LE, 45

Brusilow SW, 622

Buja LM, 610

Burdach S, 270

Burri R, 574

Carlson SE, 404

Castro R, 45

Cha C-JM, 40

Chacko A, 124

Chan JCM, 528

Chemtob S, 106

Chen F, 83, 89

Cheng PY, 202

Cheng Y, 202

Chung Y, 75

Clark S, 554

Clyman R, 616

Coates PM, 211

$\mathrm{M}, 534$

Connuck DM, 509

Cooke RE, 62

Corbet A, 190

Corbett RJT, 579

Cotton RGH, 435

Crain-Denoyelle A-M 290

Czaja JF, 26

Dabbagh S, 146
Hill JM, 95

Hindmarsh PC, 276

Hirata Y, 244

Hjalmarson O, 496, 501

Holmberg C, 327

Hoogland RA, 34

Horswill CA, 309

Hull WM, 597

Hüppi PS, 574

Huq AHMM, 11

Hurlet A, 266

Hutson SM, 281

Ibata Y, 227

Ikegami M, 518

Indo $\mathrm{Y}, 211$

Innis SM, 413

Isolauri E, 290

Isozaki-Fukuda Y, 244

Ito $\mathrm{M}, 11$

Itskovitz (Eldor) J, 381

Iwamoto HS, 158

Jackson EM, 534

Jalonen T, 290

Janssen BJA, 479

Jenkins ME, 627

Johansson BB, 473

Johnson PE, 315

Jørgensen M, 464

Kainer G, 528

Kajio F, 505

Kalhan SC, 23

Kaminsky S, 541

Kapitulnik J, 469

Karpatkin M, 266

Kattner E, 591

Katz SL, 118

Kaufman MP, 95

Kaufman P, 479

Kazui M, 396

Kelley RI, 564

Kercsmar CM, 75

Kien CL, 309, 423

Kikawa Y, 5

Kirby ML, 509

Kisker CT, 256

Klautz RJM, 375

Klitzner TS, 83, 89

Knoppel E, 362

Kobayashi T, 239

Kobayashi Y, 244

Ko F-J, 322

Kohl S, 301

Koivula T, 290

Kojima T, 244

Kollee L, 550

Komeda T, 227

Krannig G, 135

Kunjummen RD, 355

Kuribayashi T, 227

Kuroda Y, 11

LaGamma EF, 381

Lam RW, 28

Langenbeck U, 430

Lantzy A, 606

Laptook AR, 368, 579

Lazeyras F, 574

Leake RD, 45

Leatherbury L, 509
Lecce JG, 337

L'Ecuyer TJ, 232

Lelievre-Pegorier M, 450

Lemieux B, 315

Lemons JA, 423

Lewis DS, 534

Liechti-Gallati S, 304

Liechty ED, 423

Lin JJ-C, 232

Li W-Z, 239

Lloyd TR, 207

Lorenz JM, 457

Lyn-Cook LE, 337

Maechler M, 304

Maeda K, 439

Maier R, 591

Majander A, 327

Malik N, 304

Mandl KD, 444

Manuli MA, 457

Maresh M, 541

Marvin Jr WJ, 207

Massie Jr FS, 528

McCann MT, 315

McCoy KS, 309

McMahan MJ, 597

Meddings JB, 342

Merkx G, 550

Merlet-Benichou C, 450

Michaelsen KF, 464

Mistry J, 560

Mitton SG, 418

Miyagawa ST, 505

Monin P, 294

Monnens L, 550

Moore P, 62

Moore TJ, 396

Morishima M, 505

Morris M, 304

Mortola JP, 165

Moser H, 304

Mowat AP, 276

Nagaoka A, 227

Naik B, 587

Naito E, 11

Nakagawa M, 227

Nakai A, 5

Nakamichi K, 227

Nakamura T, 227

Nakano M, 528

Nakazawa M, 505

Nelson T, 248

Nguyen A, 362, 554

Noel GJ, 118

Noguchi A, 248

Nyhan WL, 15, 322

Obladen M, 591

O'Day TL, 606

O'Dorisio T, 309

Oh W, 40, 294

Olievier CN, 491

Olson AL, 256

Ono A, 244

Opleta-Madsen K, 342

Orii T, 439

O'Shea JJ, 216

Owens M, 190

Paisley JE, 351

Parrish MD, 95 
Parrott RH, 626

Pedraz C, 165

Peeples JM, 404

Peeters LLH, 479

Perkkiö M, 327

Perlman S, 256

Perman JA, 396

Pihko H, 327

Pinyerd BL, 309

Plunkett JM, 554

Polk DH, 28, 518

Ponzone A, 435

Porter JC, 368

Posse S, 574

Previs SF, 216, 439

Prodanos C, 15

Pryds O, 570

Pryhuber GS, 597

Raghavender $\mathrm{BH}, 355$

Refat M, 396

Rhodes PG, 404

Rigaux A, 193

Rinaldo P, 216, 439

Risby TH, 396

Risteli J, 276

Risteli L, 276

Robertson B, 69, 239, 591

Robillard JE, 256

Rodriguez MP, 522

Rogers S, 222

Rosenfeld CR, 368

Rossi EM, 23

Ross MG, 45

Rudolph AM, 158, 381

Ruley JR, 579

Saijo T, 11

Saito M, 5

Sandberg K, 181, 496, 501
Sariola H, 327

Sawaragi I, 244

Sawaragi S, 244

Schadewaldt P, 430

Schadow B, 591

Scheerer R, 616

Schipper IB, 375

Schmidt-Sommerfeld E, 216

Schulte D, 232

Schwartz H, 591

Schwarz KB, 396

Searls RL, 170

Segal S, 222

Segerer H, 591

Sender L, 362

Sennhauser F, 304

Shaddy RE, 351

Shaffer TH, 170

Shaul PW, 610

Shigematsu Y, 5

Shimizu N, 439

Shimoo K, 227

Sibley CP, 541

Siesjö BK, 473

Silberberg A, 181, 496, 501

Sillanaukee P, 290

Silver S, 469

Silverstein FF, 587

Simpson J, 587

Sjöqvist BA, 501

Skov L, 464, 570

Sladek M, 181

Smeets D, 550

Smith BA, 256

Smith JB, 355

Smith M, 15

Sohmer H, 469

Sokal EM, 286
Sosenko IRS, 413, 522

Speer CP, 69

Stambouly JJ, 261

Steendijk P, 375

Stevens P, 591

Stiles AD, 337

Stonestreet BS, 294

Sudo M, 5

Suen $\mathrm{Y}, 362$

Sundell HW, 181

Suomalainen A, 327

Suzuki Y, 239, 439

Sweetman FR, 15

Sweetman L, 15, 322

Swiatek KR, 331

Szeto HH, 202

Takao A, 505

Takeda E, 11

Tanaka K, 211

Tang Z, 266

Taniwaki M, 227

Teitel DF, 158, 375

Thomas CR, 541

Thomas DJ, 141

Thonney F, 304

Tolley EA, 404

Tracey BM, 560

Trivedi $\mathrm{P}, 276$

Tuchman M, 315

Ulshen MH, 337

van Bel F, 375

Van Coster RN, 1

Van den Brande JL, 34

van der Mooren K, 487

van de Ven C, 362, 554

Vanhoutte PM, 176

van Valen $F, 270$
VanWinkle WB, 135

Varma DR, 106

Vazquez RL, 606

Velvis $\mathrm{H}, 62$

Ventura GJ, 135

Verkeste CM, 479

Vettenranta K, 327

von Freeden U, 270

Waggoner DJ, 444

Wallin R, 281

Walther FJ, 518

Wang XL, 544

Warburton D, 518

Watchko JF, 606

Weaver C, 560

Welch RD, 216

Wendel U, 430

Wennberg RP, 473

Werck M-C, 100

Werkman SH, 404

Wetzel GT, 83, 89

Whitsett JA, 597

Wilcken DEL, 544

Winchurch RA, 141

Wladimiroff JW, 487

Wolff J, 322

Wolff JA, 15

Wolfson MR, 170

Wolsink JG, 491

Wu D-L, 202

Yamaguchi S, 439

Yeh Y-Y, 55

Yoshida I, 15

Zellers TM, 176

Zessack N, 270

Zinn AB, 216

Zipf TF, 135

Zipf WB, 309

Zoltoski R, 50 